\title{
Gap inversion in quasi-one-dimensional Andreev crystals
}

\author{
Mikel Rouco $\odot,{ }^{1, *}$ F. Sebastian Bergeret $\odot,{ }^{1,2, \dagger}$ and Ilya V. Tokatly $\odot^{3,4,2, \ddagger}$ \\ ${ }^{1}$ Centro de Física de Materiales (CFM-MPC), Centro Mixto CSIC-UPV/EHU, Manuel de Lardizabal 5, E-20018 San Sebastián, Spain \\ ${ }^{2}$ Donostia International Physics Center, Manuel de Lardizabal 4, E-20018 San Sebastian, Spain \\ ${ }^{3}$ Nano-Bio Spectroscopy Group, Departamento Física de Materiales, Universidad del País Vasco, Avenida Tolosa 72, \\ E-20018 San Sebastián, Spain \\ ${ }^{4}$ IKERBASQUE, Basque Foundation for Science, E-48011 Bilbao, Spain
}

(Received 10 July 2020; revised 11 September 2020; accepted 26 January 2021; published 15 February 2021)

\begin{abstract}
We study a periodic arrangement of magnetic regions in a quasi-one-dimensional superconducting wire. Due to the local exchange field, each region supports Andreev bound states that hybridize, forming Bloch bands in the subgap spectrum of what we call the Andreev crystal (AC). As an illustration, ACs with ferromagnetic and antiferromagnetic alignment of the magnetic regions are considered. We relate the spectral asymmetry index of a spin-resolved Hamiltonian to the spin polarization and identify it as the observable that quantifies the closing and reopening of the excitation gap. In particular, antiferromagnetic ACs exhibit a sequence of gapped phases separated by gapless Dirac phase boundaries. Heterojunctions between antiferromagnetic ACs in neighboring phases support spin-polarized bound states at the interface. In close analogy to the charge fractionalization in Dirac systems with a mass inversion, we find a fractionalization of the interface spin.
\end{abstract}

DOI: 10.1103/PhysRevB.103.064505

\section{INTRODUCTION}

Nonmagnetic impurities [1] in a superconductor do not substantially modify its spectrum. In contrast, a magnetic defect may lead to bound states localized around this region [2-12]. The features of such bound states depend on the size of the magnetic impurity and the strength of the exchange interaction [10]. In a quasi-one-dimensional (quasi-1D) ballistic superconducting wire [13] with a magnetic region one can distinguish two different limiting cases. In one case the magnetic exchange coupling is strong and concentrated at a pointlike impurity, resulting in the appearance of two nondegenerate states within the superconducting gap with opposite energies with respect to the Fermi energy. These are the so-called Yu-Shiba-Rusinov (YSR) states [2-4]. In the other limiting case the magnetic region has a finite size, and the exchange energy is small compared to the Fermi energy. Electrons can go through the magnetic region without being backscattered. Instead, they can be reflected as holes only via the Andreev reflection [5]. Such reflection events couple the electron and hole branches within each valley at the $\pm k_{F}$ points [see sketch in Fig. 1(a)] and induce two pairs of degenerate bound states in the superconducting gap with opposite energies $\pm \epsilon_{0}$, known as Andreev bound states. This degeneracy can be lifted if the two Fermi valleys are coupled via backscattering.

Whereas YSR states are generated by magnetic impurities of atomic size, Andreev bound states can be found in ballistic mesoscopic magnetic regions $[10,14]$. This latter case

\footnotetext{
*mikel.rouco@ehu.eus

${ }^{\dagger}$ fs.bergeret@csic.es

‡ilya.tokatly@ehu.es
}

is well described from a semiclassical perspective [15] in which electrons, crossing the magnetic region, accumulate a spin-dependent phase $e^{i \sigma \Phi}$. Here, $\Phi=\int \frac{d x}{\hbar v_{F}} h(x)$ is the phase accumulated for a collinear exchange field $h(x)$, the Fermi velocity is $v_{F}$, and $\sigma= \pm$ encodes the difference between spin-up and -down electrons. Holes accumulate the same phase, but with opposite sign, $e^{-i \sigma \Phi}$.

When several impurities form a periodic chain, it is natural to expect single-impurity bound states to hybridize, forming bands. Such bands have been widely studied in chains of magnetic atoms in the YSR limit [16-23], mainly motivated by the appearance of topological phases that may host Majorana zero modes on the endings of the wire. However, little attention has been paid to chains of semiclassical magnetic impurities that can be, for example, realized in a mesoscopic superconducting wire connected to a periodic array of ferromagnetic electrodes, as sketched in Fig. 1(b).

In this paper we study these semiclassical chains, which we denote as Andreev crystals (ACs). We focus on an AC with a collinear magnetization and analyze its spectral properties and possible quantum phases that emerge by changing the parameters of the chain. Phases are gapped and separated by gapless regions. We identify the total spin of the system as the observable which reveals these different phases. As discussed in Sec. II, from a very general perspective, the spin is determined by the asymmetry index of the spin-resolved Hamiltonian, i.e., the difference between the number of states below and above the Fermi energy. In gapped systems this index, and hence the spin, can change only by closing the gap. In Sec. III we focus on two types of ACs, ferromagnetic and antiferromagnetic chains, and determine the corresponding spectra. By changing the magnetic phase $\Phi$, we find gapped and gapless phases. In particular, antiferromagnetic ACs exhibit a sequence of 
(a)

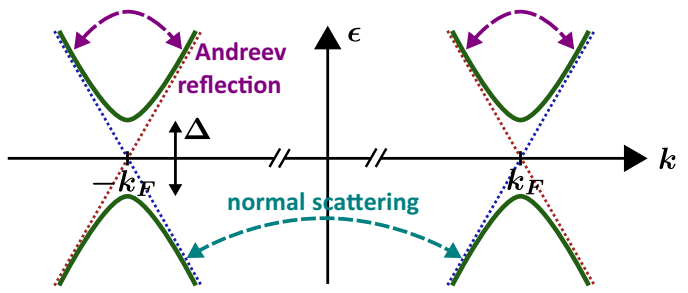

(b)

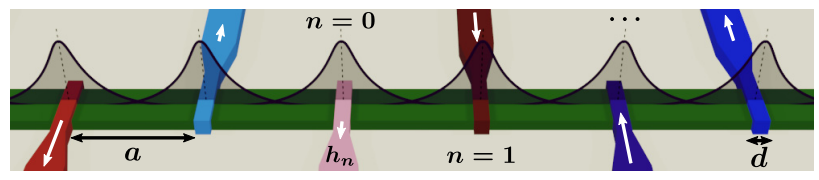

FIG. 1. (a) Schematic drawing of the spectrum of a quasi-1D $s$-wave superconductor. The electron (dashed blue line) and hole branches (dashed red line) couple, forming valleys (solid green line) and opening a gap equal to $\Delta$ at the Fermi surface. As sketched by the dashed arrows, Andreev reflections couple electron and hole branches within each valley, whereas normal backscattering events couple quasiparticles at different valleys. (b) Sketch of a possible experimental realization of an Andreev crystal: A superconducting wire (in green) is in contact with ferromagnetic fingers. The latter induce a local exchange field in the superconductor in the direction of the white arrows, strong enough to locally break the superconducting phase. The black curve above the structure represents the localized Andreev states bounded to each magnetic region which hybridize, forming the Andreev bands.

gapped phases separated, at half-integer values of $\Phi / \pi$, by gapless phase boundaries with Dirac points. In Sec. IV we show that a hybrid system with two such semi-infinite antiferromagnetic ACs may exhibit spin-polarized bound states at the interface which are similar to the states found in Dirac systems with a spatial mass inversion [24-27].

\section{SPIN POLARIZATION IN SYSTEMS WITH COLLINEAR EXCHANGE FIELDS}

We consider a quasi-1D $s$-wave superconductor [28] in the presence of a collinear exchange field, $\hat{h}(x)=\hat{\sigma}_{z} h(x)$, such that the spin along the $z$ direction is a conserved quantity (here, $\hat{\sigma}_{z}$ stands for the third Pauli matrix). The Bogoliubovde Gennes (BdG) Hamiltonian describing the system is block diagonal in spin with

$$
\hat{H}_{\sigma}(x)=\hat{\tau}_{3} \xi+\hat{\tau}_{1} \Delta(x)-\sigma h(x) .
$$

Here, $\hat{\tau}_{i=1,2,3}$ are the Pauli matrices spanning the Nambu (electron-hole) space, $\hat{\xi}$ stands for the quasiparticle energy operator, $\Delta(x)$ describes the superconducting order parameter, and $\sigma$ is the spin label [29]. From the corresponding imaginary-frequency Green's functions (GFs), $\hat{G}_{\uparrow(\downarrow)}(\epsilon)=$ $\left[i \epsilon-\hat{H}_{\uparrow(\downarrow)}\right]^{-1}$, one can compute the total spin polarization of the system at zero temperature:

$$
S=\frac{\hbar}{4} \lim _{\tau \rightarrow 0} \operatorname{Tr} \int \frac{d \epsilon}{2 \pi}\left[\hat{G}_{\uparrow}(\epsilon)-\hat{G}_{\downarrow}(\epsilon)\right] e^{i \epsilon \tau},
$$

where the trace runs over the coordinate $\otimes$ Nambu space. Since the spin-up and -down components of the Hamiltonian are related by the transformation $\hat{H}_{\downarrow}=-\hat{\tau}_{2} \hat{H}_{\uparrow} \hat{\tau}_{2}$, the GFs also fulfill $\hat{G}_{\downarrow}(\epsilon)=-\hat{\tau}_{2} \hat{G}_{\uparrow}(-\epsilon) \hat{\tau}_{2}$. Substituting this relation into
Eq. (2) and using the cyclic property of the trace, we obtain

$$
\begin{aligned}
\frac{2 S}{\hbar} & =\frac{1}{2} \lim _{\tau \rightarrow 0} \operatorname{Tr} \int \frac{d \epsilon}{2 \pi}\left[\frac{1}{i \epsilon-\hat{H}_{\uparrow}}+\frac{1}{-i \epsilon-\hat{H}_{\uparrow}}\right] e^{i \epsilon \tau} \\
& =-\frac{1}{2} \lim _{\tau \rightarrow 0} \sum_{n} \operatorname{sgn}\left(E_{n \uparrow}\right) e^{-\left|E_{n \uparrow}\right| \tau},
\end{aligned}
$$

where $E_{n \uparrow}$ stands for the energy of the $n$th eigenstate of the spin-up Hamiltonian. The expression in the last line corresponds to the difference between the number of states below and above the Fermi energy for a given spin projection, and it is known as the spectral asymmetry index, widely used in topology [30-32], quantum field theory, and condensedmatter physics [33-37]. In a gapped system, an adiabatic deformation of the Hamiltonian can change the value of this index only by closing and reopening the gap. This precisely occurs in ACs, as we discuss next.

\section{ANDREEV CRYSTALS}

We define an AC as a periodic arrangement of semiclassical magnetic regions in a superconductor. In the following we consider a quasi-1D structure of collinear magnetic regions located at the points $X_{n}=a n$ (see Fig. 2) and assume that the lateral dimensions of the system are small enough to treat each conduction channel separately and that the width of the magnetic regions is much smaller than the superconducting coherence length. The latter allows us to treat the magnetic regions in the semiclassical limit as pointlike impurities with a strength proportional to the corresponding magnetic phase $\Phi_{n}$, such that the BdG equation for a spin projection $\sigma$ reads

$$
\begin{aligned}
& {\left[-i \nu \hbar v_{F} \hat{\tau}_{3} \partial_{x}+\hat{\tau}_{1} \Delta-\sigma \hbar v_{F} \sum_{n} \Phi_{n} \delta\left(x-X_{n}\right)\right] \Psi_{\nu \sigma}(x)} \\
& \quad=\epsilon_{\nu \sigma} \Psi_{\nu \sigma}(x)
\end{aligned}
$$

where $v= \pm$ relates to the two electron-hole valleys at $\pm k_{F}$. The key feature of the semiclassical impurities is that quasiparticles do not backscatter when traversing them and only accumulate a phase,

$$
\Psi_{\sigma}\left(X_{n}^{R}\right)=e^{i \sigma \hat{\tau}_{3} \Phi_{n}} \Psi_{\sigma}\left(X_{n}^{L}\right) .
$$

Here, $\sigma$ and $\tau_{3}$ reflect the fact that the sign of the accumulated phase is different for spin-up and -down quasiparticles and for electrons and holes, respectively, and $X_{n}^{L}\left(X_{n}^{R}\right)$ stands for the position of the left (right) interface of the $n$th magnetic region. The $\delta$ functions in the first-order differential equation shown in Eq. (4) are a shorthand notation of the boundary conditions introduced by the semiclassical impurities, Eq. (5). The absence of backscattering allows separate treatment of the two Fermi valleys, so that we can drop the $v$ index for brevity.

The general solution to Eq. (4) in the region between two neighboring impurities, $X_{n}<x<X_{n+1}$, reads

$$
\Psi_{\sigma}(x)=B_{\sigma n+1}^{+} e^{\frac{x-X_{n+1}}{\xi}}|+\rangle+B_{\sigma n}^{-} e^{-\frac{x-X_{n}}{\xi}}|-\rangle .
$$

Here, $\xi \equiv \frac{\hbar v_{F}}{\sqrt{\Delta^{2}-\epsilon^{2}}}$ is the superconducting coherence length, $B_{\sigma n}^{+(-)}$is the amplitude of the contribution from the spinor that 

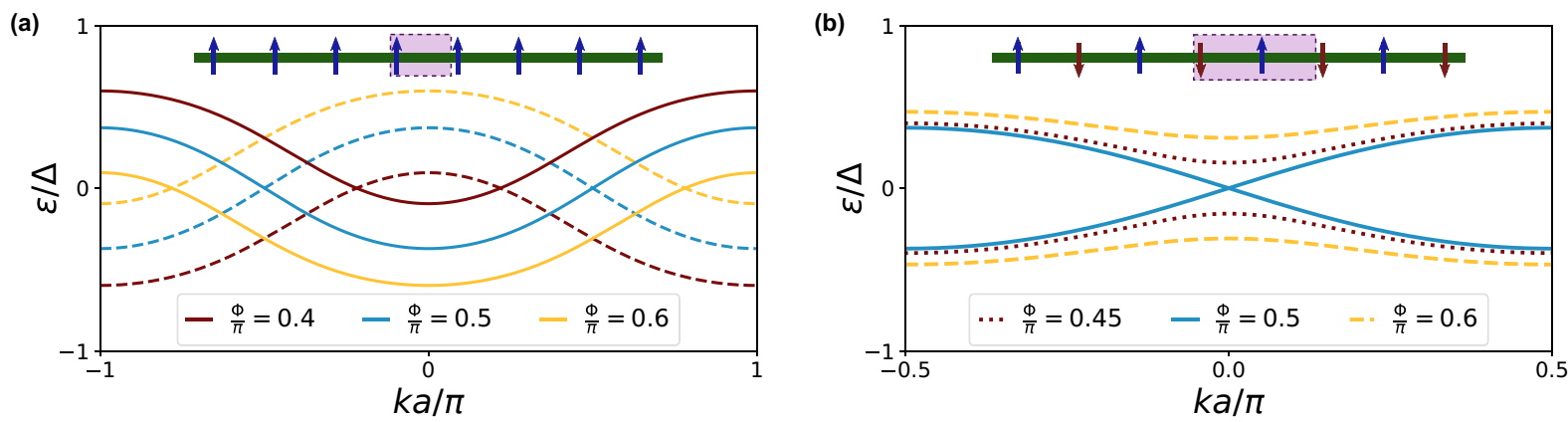

FIG. 2. Spectrum of (a) ferromagnetic and (b) antiferromagnetic Andreev crystals for energies within the superconducting gap, different values of $\Phi$, and a fixed separation between impurities given by $e^{-a / \xi}=0.2$. In (a) the solid (dashed) line corresponds to spin-up (spin-down) states. The insets are top views of the structure that show the unit cells as shaded regions.

decays from the $n$th magnetic region to the left (right), and

$$
| \pm\rangle \equiv \frac{e^{ \pm i \theta / 2}}{\sqrt{2 \cos \theta}}\left(\begin{array}{c}
1 \\
\pm i e^{\mp i \theta}
\end{array}\right)
$$

where $e^{i \theta} \equiv \frac{\sqrt{\Delta^{2}-\epsilon^{2}}+i \epsilon}{\Delta}$ is the Andreev factor. Applying the boundary conditions in Eq. (5) to this relation, we obtain the equations for the $B^{ \pm}$coefficients in Eq. (6), which can be recast into an effective tight-binding model by keeping terms up to first order in $e^{-a / \xi}$. Specifically, in the limit where $e^{-a / \xi} \ll 1$, coefficients $B^{-}$at each site $n$ can be related to $B^{+}$ as follows:

$$
B_{\sigma n}^{-} \approx i \sigma \frac{\Delta \sin \Phi_{n}}{\sqrt{\Delta^{2}-\epsilon^{2}}} B_{\sigma n}^{+},
$$

while the rescaled $B^{+}$coefficients, $b_{\sigma n} \equiv \sin \Phi_{n} B_{\sigma n}^{+}$, satisfy a tight-binding-type eigenvalue problem,

$$
\left(\sigma \omega_{\sigma}-\omega_{0 n}\right) b_{\sigma n}=t_{n+1} b_{\sigma n+1}+t_{n} b_{\sigma n-1} .
$$

Here, the effective eigenvalue $\omega_{\sigma} \equiv \frac{\epsilon_{\sigma}}{\sqrt{\Delta^{2}-\epsilon_{\sigma}^{2}}}$ is a function of the physical energy $\epsilon_{\sigma}, \omega_{0 n}=\frac{\cos \Phi_{n}}{\sin \Phi_{n}}$ is the value of $\omega_{\sigma}$ at the energy $\epsilon_{0 n}=\Delta \frac{\left|\sin \Phi_{n}\right|}{\tan \Phi_{n}}$ of the $n$th single-impurity (spinup) bound state $[5,14,15]$, and $t_{n} \equiv-\frac{e^{-a / \xi}}{\sin \Phi_{n}}$ is the hopping amplitude. The expression in Eq. (8) is valid for any AC with arbitrary distribution of collinear magnetization. Here, we focus on two cases that show rather different qualitative results: the ferromagnetic and antiferromagnetic ACs described by equal magnetic regions pointing in the same, $\Phi_{n}=\Phi$, or alternating, $\Phi_{n}=(-1)^{n} \Phi$, directions, respectively.

\section{A. Ferromagnetic ACs with $\Phi_{n}=\Phi$}

Ferromagnetic ACs are built by equal magnetic regions whose exchange field points in the same direction and are described by a unit cell containing a single magnetic region. The solution of Eq. (8) for such systems simply reads $b_{\sigma n}=$ $e^{i k n a}$ and $\omega_{\sigma}(k)=\sigma\left(\omega_{0}+2 t \cos k a\right)$, where $k$ is the Bloch momentum [38]. Hence, the physical Andreev energy bands are

$$
\frac{\epsilon_{\sigma}(k)}{\Delta}=\sigma \frac{\omega_{0}+2 t \cos k a}{\sqrt{1+\left(\omega_{0}+2 t \cos k a\right)^{2}}},
$$

where $t$ has to be evaluated at the energy of the single-impurity level $\epsilon_{0}$. In Fig. 2(a) we show the resulting energy spectrum within the Brillouin zone, $-\pi / a<k<\pi / a$, for different values of $\Phi$. It consists of two symmetric Andreev bands, one for each spin projection $\sigma$, centered at $\sigma \epsilon_{0}$. With an increase of $\Phi$ the two bands overlap but remain independent as they correspond to different spin projections. As long as there is a gap between the bands, variations of $\Phi$ do not modify the spectrum asymmetry, and thus, the spin polarization per unit cell remains unchanged [see Eq. (3)]. When the bands overlap, the spin continuously increases with the further increase of $\Phi$ until the bands pass through each other and the gap reopens. After the reopening, the total spin change is $\hbar / 2$ per Fermi valley (i.e., $\hbar$ in total).

\section{B. Antiferromagnetic ACs with $\Phi_{n}=(-1)^{n} \Phi$}

Antiferromagnetic ACs, formed by equal magnetic impurities with the direction of their exchange fields alternating between up and down along the $z$ axis, show some features of greater interest. In this case the unit cell contains two antialigned impurities [see the inset sketch in Fig. 2(b)], and it is convenient to rewrite Eq. (8) as follows:

$$
\left(\sigma \omega_{\sigma}-\hat{\Omega}_{0}\right) C_{\sigma m}=\hat{T} C_{\sigma m-1}+\hat{T}^{\dagger} C_{\sigma m+1},
$$

where now $C_{\sigma m} \equiv\left[\begin{array}{ll}b_{\sigma 2 m} & b_{\sigma 2 m+1}\end{array}\right]^{T}$ is a spinor and the matrices

$$
\hat{\Omega}_{0}=\left(\begin{array}{cc}
\omega_{0} & t \\
t & -\omega_{0}
\end{array}\right), \quad \hat{T}=\left(\begin{array}{cc}
0 & -t \\
0 & 0
\end{array}\right)
$$

correspond to the unit-cell Hamiltonian and the intercell hopping, respectively. Equation (10) describes a chain with a diatomic unit cell and the dispersion relation $\omega_{\sigma}=$ $\pm \sqrt{\omega_{0}^{2}+4 t^{2} \sin ^{2} k a}$. This dispersion relation translates into the following Andreev bands:

$$
\frac{\epsilon_{\sigma}(k)}{\Delta}= \pm \sqrt{\frac{\omega_{0}^{2}+4 t^{2} \sin ^{2} k a}{1+\omega_{0}^{2}+4 t^{2} \sin ^{2} k a}},
$$

shown in Fig. 2(b). Because the period is doubled with respect to the ferromagnetic case, the number of bands is also doubled. There are two bands per spin species which are fully symmetric with respect to the Fermi energy, and therefore, the spin polarization is zero [see Eq. (3)]. The spectrum shows 
(a)

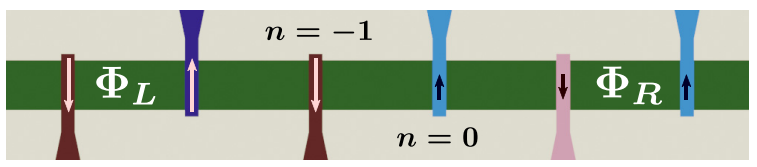

(b)

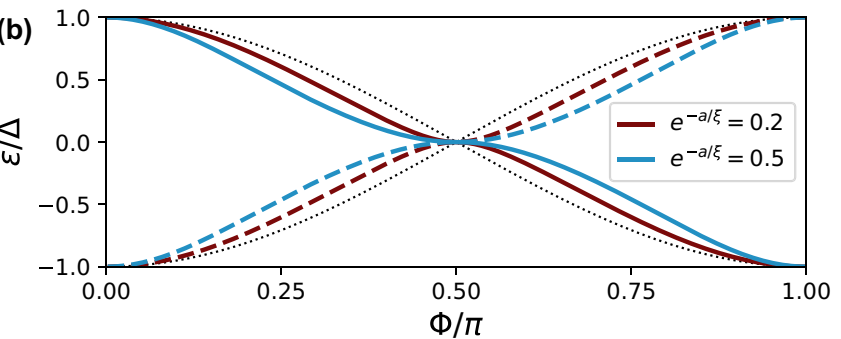

FIG. 3. (a) Sketch of a junction between two antiferromagnetic Andreev crystals. (b) Energy of the spin-up (solid lines) and spindown (dashed lines) bound states in a symmetric inverted junction, $\Phi_{R}=-\Phi_{L}=\Phi$, in terms of $\Phi$ and for different values of $e^{-a / \xi_{0}}$ [Eq. (18)]. The dotted black lines are the single impurity Andreev levels $\pm \epsilon_{0}$ that determine the gap edges

a gap equal to $2 \omega_{0}=2 \frac{\cos \Phi}{\sin \Phi}$. The gap is finite for all $\Phi$, except for half-integer values of $\Phi / \pi$, when it closes and the spectrum exhibits a Dirac point at $k_{D}=0$. In the vicinity of the critical values, $\Phi=\pi\left(l+\frac{1}{2}\right)$, where $l$ is an integer, the eigenvalue problem of Eq. (10) linearized around the Dirac point in the $k$ space reads

$$
\left(\begin{array}{cc}
\sigma \omega_{\sigma}-\omega_{0} & -2 i t k a \\
2 i t k a & \sigma \omega_{\sigma}+\omega_{0}
\end{array}\right) C_{\sigma}(k)=0,
$$

which has the form of a 1D Dirac equation with $\omega_{0}$ playing the role of the mass. The closing and reopening of the gap are associated with a sign change of the mass term (gap inversion). Interestingly, the gap can also get inverted without closing: at values of $\Phi=l \pi$ the Andreev bands merge into the continuum of the spectrum and reenter the superconducting gap in inverted order [10].

\section{INVERTED ANTIFERROMAGNETIC AC JUNCTIONS}

Various realizations of an inhomogeneous Dirac model with the mass inversion have been widely studied in quantum field theory and condensed-matter physics [24-27,36,39,40]. The most striking features of this model are the presence of bound states at the interface where the mass inversion takes place and the fractionalization of the interface charge. As we discuss next, a junction between two antiferromagnetic ACs with inverted gap is another example of such systems, but with a fractionalized interface spin instead of a charge.

To establish the analogy, we consider a junction between two semi-infinite antiferromagnetic ACs, where the separation between impurities $a$ remains constant all along the structure and the magnetic regions in the left and right crystals are described by a magnetic phase equal to $\Phi_{L}$ and $\Phi_{R}$, respectively [see the sketch in Fig. 3(a)]. Such a system is described by the tight-binding equations, Eq. (10), at each side of the junction, namely,

$$
\left(\sigma \omega_{\sigma}-\hat{\Omega}_{0 L}\right) C_{\sigma m}=\hat{T}_{m-1} C_{\sigma m-1}+\hat{T}_{m+1}^{\dagger} C_{\sigma m+1}
$$

at the left chain $(m<0)$ and

$$
\left(\sigma \omega_{\sigma}-\hat{\Omega}_{0 R}\right) C_{\sigma m}=\hat{T}_{m-1} C_{\sigma m-1}+\hat{T}_{m+1}^{\dagger} C_{\sigma m+1}
$$

at the right chain $(m \geqslant 0)$. Here, $\hat{\Omega}_{0 L}\left(\hat{\Omega}_{0 R}\right)$ stands for the expression of $\hat{\Omega}_{0}$ in Eq. (11) with $\Phi=\Phi_{L}\left(\Phi=\Phi_{R}\right)$. We look for bound states, i.e., solutions that decay as $C_{\sigma m}=C_{\sigma}^{L} e^{m \kappa_{\sigma}^{L}}$ into the left crystal and as $C_{\sigma m}=C_{\sigma}^{R} e^{-m \kappa_{\sigma}^{R}}$ into the right one, where the decay is determined by the positive-real-part complex number $\kappa_{\sigma}^{L(R)}$. From Eqs. (14) and (15) we find that

$$
\sinh \frac{\kappa_{\sigma}^{L(R)}}{2}=\frac{\sqrt{\omega_{0 L(R)}^{2}-\omega_{\sigma}^{2}}}{2\left|t_{L(R)}\right|},
$$

where $t_{L}=t_{m<0}$ and $t_{R}=t_{m \geqslant 0}$, and that the bound state exists only when the equation

$$
\frac{\sigma \omega_{\sigma}-\omega_{0 L}}{\sqrt{\omega_{0 L}^{2}-\omega_{\sigma}^{2}}} e^{-\frac{\kappa_{\sigma}^{L}}{2}}=-\frac{\sigma \omega_{\sigma}-\omega_{0 R}}{\sqrt{\omega_{0 R}^{2}-\omega_{\sigma}^{2}}} e^{\frac{\kappa_{\sigma}^{R}}{2}}
$$

is fulfilled. According to Eq. (16) a bound state exists only if $\left|\omega_{\sigma}\right|<\left|\omega_{0 L}\right|$ and $\left|\omega_{\sigma}\right|<\left|\omega_{0 R}\right|$ at the same time. This implies that Eq. (17) has a solution only in inverted junctions with $\operatorname{sgn}\left(\omega_{0 R}\right)=-\operatorname{sgn}\left(\omega_{0 L}\right)$. The solution is especially simple when $\omega_{0 R}=-\omega_{0 L} \equiv \omega_{0}$ and reads $\omega_{\sigma}=$ $\sigma \operatorname{sgn}\left(\omega_{0}\right)\left(\sqrt{\omega_{0}^{2}+t^{2}}-|t|\right)$. This gives the following physical energy of the bound state:

$$
\frac{\epsilon_{\sigma}}{\Delta}=\sigma \operatorname{sgn}\left(\omega_{0}\right) \frac{\sqrt{\omega_{0}^{2}-t^{2}}-|t|}{\sqrt{1+\left(\sqrt{\omega_{0}^{2}-t^{2}}-|t|\right)^{2}}} .
$$

In Fig. 3(b) we show the bound states for both spin projections as a function of $\Phi$ for different values of $-t \sin \Phi=e^{-a / \xi}$. Near the inversion point $\left|\omega_{0} / t\right| \rightarrow 0$, the bound states are almost degenerate approaching zero energy, $\epsilon_{\sigma} \rightarrow 0$. This is reminiscent of a zero mode in a continuum 1D Dirac model with mass inversion [24]. As the bandwidth becomes comparable to $\omega_{0}$, the states split, forming a symmetric pair of levels in the gap between the Andreev bands.

To calculate the spin $S$ induced at the contact between the two semi-infinite antiferromagnetic ACs, we average over all possible terminations of the chains. This is equivalent to the so-called sliding window average method (see, for example, Sec. 4.5 of Ref. [41]), used to compute the surface charge density by averaging over all possible unit cell choices. The calculation is specially simple in the limit when the single-impurity Andreev states are decoupled from each other, $e^{-a / \xi} \ll 1$. In a previous work [10], we show that the spin polarization of a single semiclassical magnetic impurity of magnetic phase $\Phi$ in a quasi-1D superconducting wire is $2 S_{0}(\Phi) / \hbar=2\left[\left(\Phi+\frac{\pi}{2}\right) \bmod \pi\right]$, where $\bmod$ stands for the modulo operation and accounts for a jump by two electronic spins every time the single-impurity levels cross the Fermi energy. In Fig. 4(a) we show the staircase shape of $S_{0}(\Phi)$ in terms of the magnetic phase for a single semiclassical impurity. We now consider the junction between the two antiferromagnetic ACs. It has four possible ending configurations: both chains have the same number of up and down magnetic impurities, the right (left) Andreev chain has an extra 
(a)

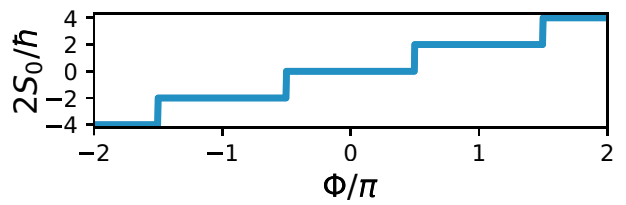

(b)

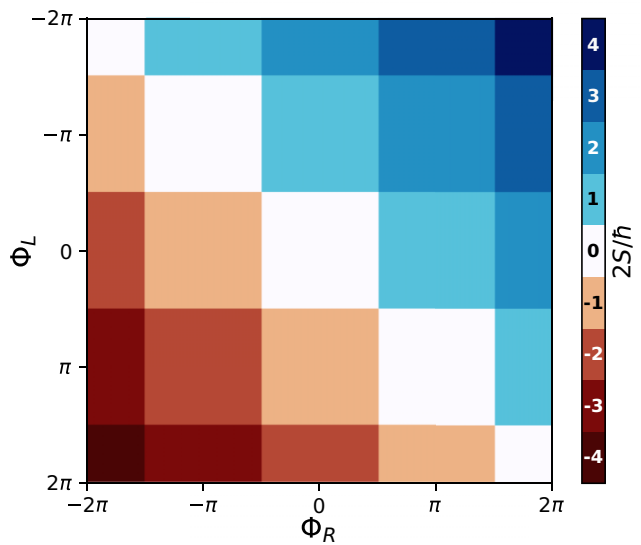

FIG. 4. (a) Total spin polarization of the single-impurity system in terms of $\Phi$. (b) Spin polarization of the junction between two antiferromagnetic Andreev chains as a function of $\Phi_{L}$ and $\Phi_{R}$. It is calculated from Eq. (19), with $S_{0}\left(\Phi_{L(R)}\right)$ from (a).

up (down) magnetic region, or both chains are unbalanced. Consequently, the total spin polarization of the junction, calculated from the average over the four possible configurations, reads

$$
S=\frac{S_{0}\left(\Phi_{R}\right)-S_{0}\left(\Phi_{L}\right)}{2} .
$$

Starting from the uncoupled impurities, if one adiabatically switches on the coupling, the Andreev bands start widening. However, in the considered configuration the gap never closes, and as we discussed after Eq. (3), the spin cannot change and is hence given by Eq. (19). In Fig. 4(b) we show the total spin of the junction in terms of $\Phi_{L}$ and $\Phi_{R}$. Interestingly, the spin polarization can now be equal to an odd integer times the electronic unit, in contrast to the always even value of $S_{0}(\Phi)$. By construction, the half-integer spin (per Fermi valley) is localized at the junction between ACs. In other words, there is a fractionalization of the interface spin. Such fractionalization is a local effect. In a finite system the contribution from the edges will always lead to a total integer spin per Fermi valley. Notice that changes in the spin polarization of ACs is determined by the change in the spectral asymmetry index, Eq. (3), and hence, Eq. (19) is valid beyond the nearestneighbor tight-binding approximation. This is, indeed, confirmed by the exact numerical solution of Eq. (4) [42]

\section{CONCLUSIONS}

In this work we showed that the spin polarization of a gapped system with collinear magnetization can change only upon gap closing. This occurs in Andreev crystals for which we presented a complete study of their spectral properties for ferromagnetic and antiferromagnetic configurations. The spectrum of antiferromagnetic ACs presents a gap that remains open except for half-integer values of the magnetic phase $\Phi / \pi$, where a Dirac point is formed. We showed that junctions between antiferromagnetic ACs with inverted gaps exhibit interfacial bound states and fractionalization of the surface spin polarization. We proposed realization of these structures using, for example, a conventional superconducting wire in contact with ferromagnetic fingers such that a strong periodic exchange field, $h \gg \Delta$, is induced in the superconductor [see Fig. 1(b)]. The fingers may be made of ferromagnetic metals [43], like $\mathrm{Co}$ or $\mathrm{Ni}$, or ferromagnetic insulators, like EuS or EuO. The spectrum, and in particular the bound states formed at the interface between two antiferromagnetic chains, can be measured by a local tunneling probe, as, for example, done in Ref. [44]. In the case of a magnetic probe one can also determine the spin polarization of such states.

\section{ACKNOWLEDGMENTS}

We acknowledge funding from the Spanish Ministerio de Ciencia, Innovación y Universidades (MICINN; Projects No. FIS2016-79464-P and No. FIS2017-82804-P). I.V.T. acknowledges support from Grupos Consolidados UPV/EHU del Gobierno Vasco (Grant No. IT1249-19). The work of F.S.B. is partially funded by the European Research Council under EU's Horizon 2020 research and innovation program under Grant Agreement No. 800923 (SUPERTED).
[1] Regions of much smaller size than the superconducting coherence length $\xi_{0}$.

[2] L. Yu, Acta Phys. Sin 21, 75 (1965).

[3] H. Shiba, Prog. Theor. Phys. 40, 435 (1968).

[4] A. Rusinov, Zh. Eksp. Teor. Fiz. Pis'ma Red. 9, 146 (1968) [Sov. Phys. JETP 9, 85 (1969)] .

[5] A. Andreev, Zh. Eksp. Teor. Fiz. 49 (1966) [Sov. Phys. JETP 22, 455 (1966)].

[6] A. Sakurai, Prog. Theor. Phys. 44, 1472 (1970).

[7] A. Yazdani, Science 275, 1767 (1997).

[8] A. V. Balatsky, I. Vekhter, and J.-X. Zhu, Rev. Mod. Phys. 78, 373 (2006).
[9] K. J. Franke, G. Schulze, and J. I. Pascual, Science 332, 940 (2011).

[10] M. Rouco, I. V. Tokatly, and F. S. Bergeret, Phys. Rev. B 99, 094514 (2019).

[11] B. W. Heinrich, J. I. Pascual, and K. J. Franke, Prog. Surf. Sci. 93, 1 (2018).

[12] L. Farinacci, G. Ahmadi, G. Reecht, M. Ruby, N. Bogdanoff, O. Peters, B. W. Heinrich, F. von Oppen, and K. J. Franke, Phys. Rev. Lett. 121, 196803 (2018).

[13] A wire whose lateral dimensions are much smaller than the superconducting coherence length.

[14] F. Konschelle, I. V. Tokatly, and F. S. Bergeret, Phys. Rev. B 94, 014515 (2016). 
[15] F. Konschelle, F. S. Bergeret, and I. V. Tokatly, Phys. Rev. Lett. 116, 237002 (2016).

[16] S. Nadj-Perge, I. K. Drozdov, B. A. Bernevig, and A. Yazdani, Phys. Rev. B 88, 020407(R) (2013).

[17] F. Pientka, L. I. Glazman, and F. von Oppen, Phys. Rev. B 88, 155420 (2013).

[18] A. Heimes, P. Kotetes, and G. Schön, Phys. Rev. B 90, 060507(R) (2014).

[19] K. Pöyhönen, A. Westström, J. Röntynen, and T. Ojanen, Phys. Rev. B 89, 115109 (2014).

[20] A. Westström, K. Pöyhönen, and T. Ojanen, Phys. Rev. B 91, 064502 (2015).

[21] F. Pientka, Y. Peng, L. Glazman, and F. v. Oppen, Phys. Scr. T164, 014008 (2015).

[22] P. M. R. Brydon, S. Das Sarma, H.-Y. Hui, and J. D. Sau, Phys. Rev. B 91, 064505 (2015).

[23] M. Schecter, K. Flensberg, M. H. Christensen, B. M. Andersen, and J. Paaske, Phys. Rev. B 93, 140503 (2016).

[24] R. Jackiw and C. Rebbi, Phys. Rev. D 13, 3398 (1976).

[25] W. P. Su, J. R. Schrieffer, and A. J. Heeger, Phys. Rev. B 22, 2099 (1980).

[26] W. P. Su, J. R. Schrieffer, and A. J. Heeger, Phys. Rev. Lett. 42, 1698 (1979).

[27] B. Volkov and O. Pankratov, JETP Lett. 42, 178 (1985).

[28] We focus on the one-dimensional case to be consistent with the rest of the paper, but one can derive the expression for the total spin of a system of any dimensions following the same lines.
[29] The spin label for spin up (down) is substituted by $\sigma=\uparrow(\downarrow)$ when it appears as a subscript, whereas it takes values of $\sigma=$ $+(-)$ when it is part of an equation.

[30] M. F. Atiyah, V. K. Patodi, and I. M. Singer, Math. Proc. Cambridge Philos. Soc. 77, 43 (1976).

[31] M. F. Atiyah, V. K. Patodi, and I. M. Singer, Math. Proc. Cambridge Philos. Soc. 78, 405 (1975).

[32] M. F. Atiyah, V. K. Patodi, and I. M. Singer, Math. Proc. Cambridge Philos. Soc. 79, 71 (1976).

[33] A. J. Niemi and G. W. Semenoff, Phys. Rev. D 30, 809 (1984).

[34] R. Blankenbecler and D. Boyanovsky, Phys. Rev. D 31, 2089 (1985).

[35] A. A. Andrianov, V. A. Andrianov, V. Y. Novozhilov, and Y. V. Novozhilov, Lett. Math. Phys. 11, 217 (1986).

[36] M. Stone, Phys. Rev. B 31, 6112 (1985).

[37] G. E. Volovik, The Universe in a Helium Droplet, International Series of Monographs on Physics Vol. 117 (Oxford University Press, New York, 2003).

[38] N. Ashcroft and D. Mermin, Solid State Physics (Cengage Learning, Andover, UK, 1976).

[39] J. Goldstone and F. Wilczek, Phys. Rev. Lett. 47, 986 (1981).

[40] R. MacKenzie and F. Wilczek, Phys. Rev. D 30, 2194 (1984).

[41] D. Vanderbilt, Berry Phases in Electronic Structure Theory (Cambridge University Press, Cambridge, 2018).

[42] M. Rouco, F. S. Bergeret and I. V. Tokatly (unpublished).

[43] D. Beckmann, H. B. Weber, and H. v. Löhneysen, Phys. Rev. Lett. 93197003 (2004).

[44] J.-D. Pillet, C. H. L. Quay, P. Morfin, C. Bena, A. L. Yeyati, and P. Joyez, Nat. Phys. 6, 965 (2010). 